\title{
Bone turnover marker monitoring in osteoporosis treatment response
}

\author{
Rui Zhen Tan', Tze Ping Loh' ${ }^{2}$ and Samuel Vasikaran ${ }^{3}$
}

${ }^{1}$ Engineering Cluster, Singapore Institute of Technology, Singapore, Singapore, ${ }^{2}$ Department of Laboratory Medicine, National University Hospital, Singapore, Singapore, and ${ }^{3}$ Department of Clinical Biochemistry, PathWest-Royal Perth Hospital, Perth, Western Australia, Australia

Correspondence should be addressed to T P Loh

Email

tploh@hotmail.com
Recently, the International Osteoporosis Foundation and European Calcified Tissue Society (IOF-ECTS) working group produced recommendations for the screening of adherence to oral bisphosphonates using bone turnover markers with clinical decision limits (1). The guideline recommended that adherence can be monitored using the least significant change in bone turnover markers or using the geometric mean of reference intervals of premenopausal women. The recommendations are largely based on the findings of the TRIO study, which was a parallel, randomised controlled intervention trial comparing three orally administered bisphosphonates over a 2-year period (2). Of note, the recommendation to use the least significant change as a screening tool for adherence by the IOF-ECTS is not based on the patients who were objectively proven to be compliant throughout the initial 13 weeks of treatment. Rather, the least significant change was calculated based on all patients who were treated in the TRIO study.

The Endocrine Society (https://www.endocrine.org/) also recently published a guideline for management of osteoporosis. The guideline noted that bone turnover markers may be used as an alternative to bone mineral density scan for treatment monitoring and referenced the evidence produced by the TRIO study (3). The recommended retesting interval for bone mineral density scan at the spine and hip is every 1 to 3 years, as it takes longer for the bone mineral density to change significantly. Moreover, there is no clear numerical thresholds to indicate good response or adherence. By contrast, the change in bone turnover marker is more rapid. The Endocrine Society did not recommend specific bone turnover marker targets.
In the TRIO study, bone turnover markers including C-telopeptide of type I collagen (CTX), N-mid osteocalcin, intact pro-collagen I N-propeptide (PINP), bone alkaline phosphatase and urinary N-telopeptide of type I collagen (expressed as a ratio to urinary creatinine) were measured before treatment and at baseline 1 (a week before), baseline 2 (week 0 ) and then at 1, 2, 4, 12, 13, 48 and 96 weeks after initiation of oral bisphosphonates (2). The least significant change (also known as critical difference or reference change value), defined as the smallest difference between sequential laboratory results which is associated with a true change in the patient (4), of bone turnover markers in patients receiving oral bisphosphonates were derived from a pair of measurements take at weeks 12 and 13 after initiation of treatment. Additionally, the study also reported the reference intervals of the bone turnover markers for pre-menopausal women.

However, we contend that the biological variation concept was incorrectly applied in the TRIO study to derive the least significant change. Moreover, we demonstrate that the choice of using the mid-point of reference interval as target for response is neither specific nor sensitive in identifying patients who responded to treatment. There is a need to interpret the results of the TRIO study correctly and revise the monitoring criteria for bone turnover markers to avoid suboptimal clinical management.

The least significant change is mathematically represented by $Z \times \sqrt{ }\left(2 \times\left(C V i^{2}+C V a^{2}\right)\right)$, where $C V i$ is the within-person biological variation and $C V a$ is the laboratory analytical variation, and $Z$ is the $Z$-value that is associated with a desired probability of a true unior bidirectional change (4). Traditionally, the $Z \times \sqrt{ } 22$

Published by Bioscientifica Ltd. 
is abbreviated to a coefficient of 2.77 for calculation of a bidirectional least significant change with a $95 \%$ probability using a $z$-value of 1.96 . The least significant change defined in the TRIO study, derived following log-transformation of the data, contained the CVi and $C V a$. The $z$-value chosen (i.e. 1.96) was for bidirectional change (i.e. increase or decrease), where the direction of change cannot be predicted. As a result, the calculated least significant change values were erroneously large. In practice, because the direction of change for patients treated with oral bisphosphonate is known (a decrease since the reason for monitoring anti-resorptive therapy is to demonstrate treatment effect which is confirmed by a significant reduction in bone turnover markers), a $Z$-value of 1.65 is the correct number to apply in order to calculate the true decrease in bone turnover markers with 95\% probability. Application of a $z$-value of 1.96 leads to falsely increased least significant change values.

When calculating the least significant change, it is more appropriate to use the CVi of healthy subjects instead of patients who are on oral bisphosphonate, as the former are more similar to untreated (poorly adherent) subjects. In other words, a post-treatment response that exceeds the physiological fluctuation of an untreated subject is likely to be indicative of a response (or adherence) to treatment. The biological variation of the bone turnover marker has been described in healthy subjects and summarised previously $(5,6)$ (https://www.westgard.com/biodatabase2014-update.htm). They are used here for the revised calculations below. It is important to note that using the least significant change value derived from adherent patients will misclassify $2.5 \%$ of adherent patients, and not non-adherent/untreated subjects, as described in the IOF-ECTS guideline (1).

Moreover, the $\mathrm{CVa}$ for bone turnover markers is dependent on the laboratory methods used (7), which also limits the generalisability of least significant change value that incorporated the $\mathrm{CVa}$ of the laboratory used in the TRIO study. The least significant changes using the revised $z$-value (1.65) and $C V i$ from health individuals stratified by several $\mathrm{CVa}$ are summarised in Table 1 . Here, the reanalysis is focused on CTX and PINP, as they are recommended by the IOF and the International Federation of Clinical Chemistry and Laboratory Medicine (8). It is important to note that these values are associated with a $5 \%$ probability of classifying an untreated subject as having a true reduction that exceeded the least significant change (i.e. the false positive rate) (4). This table helps guide individual centers in choosing the least significant
Table 1 Original least significant change of CTX and PINP described in the TRIO study and the revised least significant change (LSC) using a $z$-value of 1.65 for unidirectional change (decrease) for various laboratory analytical variation (CVa).

\begin{tabular}{|c|c|c|c|c|}
\hline $\begin{array}{l}\text { Bone turnover } \\
\text { marker }\end{array}$ & $\begin{array}{l}\text { TRIO study } \\
\text { LSC, \% }\end{array}$ & $c V i, \%$ & $C V a, \%$ & Revised LSC, \% \\
\hline \multirow[t]{5}{*}{ CTX } & 56 & 10.6 & 5 & 27 \\
\hline & & 10.6 & 7 & 30 \\
\hline & & 10.6 & 10 & 34 \\
\hline & & 10.6 & 12 & 37 \\
\hline & & 10.6 & 15 & 43 \\
\hline \multirow[t]{5}{*}{ PINP } & 38 & 3.7 & 5 & 14 \\
\hline & & 3.7 & 7 & 18 \\
\hline & & 3.7 & 10 & 25 \\
\hline & & 3.7 & 12 & 29 \\
\hline & & 3.7 & 15 & 36 \\
\hline
\end{tabular}

LSC $=Z \times \sqrt{ }\left(2 \times\left(C V i^{2}+C V a^{2}\right)\right)$, where $C v i=$ within-subject biological variation.

change that best reflects the performance of the laboratory method in use.

Using the table, the revised least significant change for CTX and PINP for the analytical methods (both with $\mathrm{CVa} \sim 7 \%$ ) used in the TRIO would be $30 \%$ and $18 \%$, respectively. They are considerably smaller than the original least significant change. When the revised least significant changes were applied to the TRIO study population (based on Fig. 2 of Ref. (2)), the responder rate became:

CTX: ibandronate 98\% (48/49), alendronate 100\% $(50 / 50)$ and risedronate $94 \%(44 / 47)$ compared to previously 84, 98 and 78\%, respectively.

PINP: ibandronate $100 \% \quad(50 / 50)$, alendronate $98 \%(49 / 50)$ and risedronate 96\% (45/47) compared to previously 94,82 and $75 \%$, respectively.

Taken together, the revised least significant change value would lead to a specificity (true negative rate) of 95\% and sensitivity (true positive rate) ranging from 94\%-100\%, based on the TRIO data. The high sensitivity achieved using the revised (narrower) $z$-value and CVi fits the clinical utility of a screening test.

There are several important considerations in the methods described. First, it is assumed that the biological variation of the bone markers in healthy subjects are similar to post-menopausal women. The validity of this assumption deserves further research. Secondly, care must be exercised when interpreting an increase in the bone markers. Clinicians should consider other incident diseases that may influence bone turnover rate (e.g. recent fracture), before ascribing the laboratory observation as poor adherence. It must be emphasised that interpretation 
of bone turnover marker is a nuanced clinical judgment that should be supplemented by appropriate clinical history taking and examination and not a statistical exercise. The reader is referred to an excellent review on this topic (9). Finally, analytical variability due to change in the series of the kit for the assay of a bone marker is an important consideration. Clinicians are encouraged to select the $\mathrm{CVa}$ that best describes the laboratory assay in use in their practice to select the optimal least significant change.

In summary, the measurement of bone turnover markers can offer a convenient and responsive way to monitor the adherence of patients prescribed with oral bisphophonates. However, care must be exercised when selecting the appropriate criteria of result interpretation for optimal clinical decision making. It should be emphasised that, ideally, bone turnover marker should be measured at baseline before commencement of treatment to allow for calculation of the change with treatment.

\section{Declaration of interest}

The authors declare that there is no conflict of interest that could be perceived as prejudicing the impartiality of this commentary.

\section{Funding}

This work did not receive any specific grant from any funding agency in the public, commercial or not-for-profit sector.

\section{References}

1 Diez-Perez A, Naylor KE, Abrahamsen B, Agnusdei D, Brandi ML, Cooper C, Dennison E, Eriksen EF, Gold DT, Guañabens N et al. International Osteoporosis Foundation and European Calcified
Tissue Society Working Group. Recommendations for the screening of adherence to oral bisphosphonates. Osteoporosis International 2017 28 767-774. (https://doi.org/10.1007/s00198-017-3906-6)

2 Naylor KE, Jacques RM, Paggiosi M, Gossiel F, Peel NF, McCloskey EV, Walsh JS \& Eastell R. Response of bone turnover markers to three oral bisphosphonate therapies in postmenopausal osteoporosis: the Trio study. Osteoporosis International 201627 21-31. (https://doi. org/10.1007/s00198-015-3145-7)

3 Kanis JA, Cooper C, Rizzoli R, Reginster JY \& Scientific Advisory Board of the European Society for Clinical and Economic Aspects of Osteoporosis (ESCEO) and the Committees of Scientific Advisors and National Societies of the International Osteoporosis Foundation (IOF). European guidance for the diagnosis and management of osteoporosis in postmenopausal women. Osteoporosis International 201930 3-44. (https://doi.org/10.1007/s00198-018-4704-5)

4 Fraser CG. Changes in serial results. In Biological Variation: From Principles to Practice, pp. 67-90. Washington: Association for the Aid of Crippled Children Press, 2001.

5 Ricós C, Alvarez V, Cava F, García-Lario JV, Hernández A, Jiménez CV, Minchinela J, Perich C \& Simón M. Current databases on biological variation: pros, cons and progress. Scandinavian Journal of Clinical and Laboratory Investigation 199959 491-500. (https://doi. org/10.1080/00365519950185229)

6 Perich C, Minchinela J, Ricós C, Fernández-Calle P, Alvarez V, Doménech MV, Simón M, Biosca C, Boned B, García-Lario JV et al. Biological variation database: structure and criteria used for generation and update. Clinical Chemistry and Laboratory Medicine 201553 299-305. (https://doi.org/10.1515/cclm-2014-0739)

7 Vasikaran S, Eastell R, Bruyère O, Foldes AJ, Garnero P, Griesmacher A, McClung M, Morris HA, Silverman S, Trenti T et al. Markers of bone turnover for the prediction of fracture risk and monitoring of osteoporosis treatment: a need for international reference standards. Osteoporosis International 201122 391-420. (https://doi.org/10.1007/s00198-010-1501-1)

8 Vasikaran S, Cooper C, Eastell R, Griesmacher A, Morris HA, Trenti T \& Kanis JA. International Osteoporosis Foundation and International Federation of Clinical Chemistry and Laboratory Medicine position on bone marker standards in osteoporosis. Clinical Chemistry and Laboratory Medicine 201149 1271-1274. (https://doi.org/10.1515/ CCLM.2011.602)

9 Eastell R, Pigott T, Gossiel F, Naylor KE, Walsh JS \& Peel NFA. DIAGNOSIS OF ENDOCRINE DISEASE: Bone turnover markers: are they clinically useful? European Journal of Endocrinology 2018178 R19-R31. (https://doi.org/10.1530/EJE-17-0585)

Received 28 November 2019

Revised version received 25 February 2020

Accepted 20 April 2020 